\title{
Search for Light Higgs and Dark Photons at BaBar and Belle
}

\author{
Ming-Chuan Chang ${ }^{* \dagger}$ \\ Department of Physics, \\ Fu-Jen Catholic University, Taiwan \\ E-mail: $068190 @$ mail. fju.edu.tw
}

From supersymmetry to dark matter, many extensions of the Standard Model include the possibility of light new physics. The ideal tools to explore such theories are low-energy high-luminosity collider experiments such as $B$ factories. This report summarizes recent searches for light new physics by the $\mathrm{BaBar}$ and Belle experiments.

The European Physical Society Conference on High Energy Physics -EPS-HEP2013

18-24 July 2013

Stockholm, Sweden

${ }^{*}$ Speaker.
†On behalf of the Belle Collaboration 


\begin{tabular}{|c|cc|c|}
\hline \hline$\sqrt{s}$ & BaBar & Belle & Total \\
\hline$\Upsilon(5 S)$ & - & 121 & 121 \\
$\Upsilon(4 S)$ & 433 & 711 & 1,144 \\
$\Upsilon(3 S)$ & 30 & 3 & 33 \\
$\Upsilon(2 S)$ & 15 & 25 & 40 \\
$\Upsilon(1 S)$ & - & 6 & 6 \\
\hline Off-resonance & 54 & 94 & 138 \\
\hline \hline
\end{tabular}

Table 1: Integrated luminosities $\left(\mathrm{fb}^{-1}\right)$ collected by the $B$ factories at different center-of-mass energies. The off-resonance data were collected about $40 \mathrm{MeV}$ below the $\Upsilon(4 S)$ resonance at BABAR and at a similar offset for the $\Upsilon(4 S)$ and $\Upsilon(5 S)$ resonances in the case of Belle.

\section{Introduction}

During the last decade, the BaBar Collaboration at PEP-II [1] and the Belle Collaboration at KEKB [2][3] have respectively collected about $550 \mathrm{fb}^{-1}$ and more than $1 \mathrm{ab}^{-1}$ of data at several $\Upsilon$ resonances, mostly the $\Upsilon(4 S)$ resonance(see Table 1$)$.

\section{Search for Light $C P$-Odd Higgs boson in $\Upsilon$ decays}

The Higgs mechanism attempts to explain the origin of mass within the Standard Model (SM) [4]. Recently, experimental evidence suggests a Higgs-like states with the mass of about 125 $\mathrm{GeV}$ [5]. Several extensions of SM, such as the next-to-minimal supersymmetric Model (nMSSM) predicts more Higgs-like state [6].

The nMSSM suggests a Higgs sector contains seven states, two charged Higgs bosons, three neutral CP-even bosons, and two CP-odd bosons. The lightest CP-odd state, $A^{0}$, could be with the mass below the $b \bar{b}$ production threshold. Such a particle could be produced in $\Upsilon \rightarrow \gamma A^{0}$ decays [7] with a branching fraction as large as $10^{-4}$ for the narrow states $\Upsilon(n S)$ (where $\mathrm{n} \leq 3$ ). The predicted branching fraction depends on the $A^{0}$ mass and couplings [6]. The $A^{0}$ is accessible and well above the sensitivity of $B$-factories.

$e^{+} e^{-} \rightarrow \Upsilon(1 S, 2 S, 3 S) \rightarrow \gamma A^{0}, A^{0} \rightarrow \tau^{+} \tau^{-}$

The two taus of the $\Upsilon(n S) \rightarrow \gamma A^{0}, A^{0} \rightarrow \tau^{+} \tau^{-}$decays are identified through their leptonic decays, $\tau^{+} \rightarrow \mu^{+} v_{\tau} \bar{v}_{\mu}$ and $\tau^{-} \rightarrow e^{-} v_{\tau} \bar{v}_{e}$. The signal event should consist of exactly two oppositelycharged tracks, identified as muons or electrons. The signal yield is extracted as a function of $m_{A^{0}}$ by a simultaneous fit to the photon energy distribution of the $e e \gamma, \mu \mu \gamma$ and $e \mu \gamma$ samples. No excess is seen. The 90\% CL limits on the branching fraction are listed in Table 2 [8]. The Belle results are preliminary.

$e^{+} e^{-} \rightarrow \Upsilon(2 S) \rightarrow \Upsilon(1 S) \pi^{+} \pi^{-}, \Upsilon(1 S) \rightarrow \gamma A^{0}, A^{0} \rightarrow \tau^{+} \tau^{-}$

In this analysis, the $\Upsilon(1 S)$ resonance is produced from the $\Upsilon(2 S)$ resonance with the emission of two charged pions. The $\Upsilon(1 S)$ is identified by the dipion transition. The signal events are 


\begin{tabular}{|c|c|cc|}
\hline \hline Mode & Mass range $(\mathrm{GeV})$ & \multicolumn{2}{|c|}{ BF upper limit (90\% CL) } \\
& & BaBar & Belle \\
\hline$\Upsilon(1 S) \rightarrow \gamma A^{0}, A^{0} \rightarrow \tau^{+} \tau^{-}$ & $3.6<m_{A^{0}}<9.3$ & - & $(0.4-4.5) \times 10^{-5}$ \\
$\Upsilon(2 S) \rightarrow \gamma A^{0}, A^{0} \rightarrow \tau^{+} \tau^{-}$ & $4.16<m_{A^{0}}<9.19$ & - & $(1.61-12.17) \times 10^{-5}$ \\
$\Upsilon(3 S) \rightarrow \gamma A^{0}, A^{0} \rightarrow \tau^{+} \tau^{-}$ & $4.03<m_{A^{0}}<10.1$ & $(1.5-16) \times 10^{-5}$ & - \\
\hline \hline
\end{tabular}

Table 2: Results of light Higgs boson searches, $e^{+} e^{-} \rightarrow \Upsilon(1 S, 2 S, 3 S) \rightarrow \gamma A^{0}, A^{0} \rightarrow \tau^{+} \tau^{-}$, studied by the BaBar [8] and Belle Collaboration. The Belle results are preliminary.

\begin{tabular}{|c|c|cc|}
\hline \hline Mode & Mass range $(\mathrm{GeV})$ & \multicolumn{2}{|c|}{ BF upper limit (90\% CL) } \\
& & BaBar & Belle \\
\hline$\Upsilon(1 S) \rightarrow \gamma A^{0}, A^{0} \rightarrow \tau^{+} \tau^{-}$ & $3.5<m_{A^{0}}<9.2$ & $(0.9-13) \times 10^{-5}$ & - \\
$\Upsilon(1 S) \rightarrow \gamma A^{0}, A^{0} \rightarrow \tau^{+} \tau^{-}$ & $3.84<m_{A^{0}}<9.16$ & - & $(0.91-45.37) \times 10^{-5}$ \\
\hline \hline
\end{tabular}

Table 3: Results of light Higgs boson searches, $e^{+} e^{-} \rightarrow \Upsilon(2 S) \rightarrow \Upsilon(1 S) \pi^{+} \pi^{-}, \Upsilon(1 S) \rightarrow \gamma A^{0}, A^{0} \rightarrow \tau^{+} \tau^{-}$, studied by the BaBar [9] and Belle Collaboration. The Belle result is preliminary.

\begin{tabular}{|c|c|cc|}
\hline \hline Mode & Mass range $(\mathrm{GeV})$ & \multicolumn{2}{|c|}{ BF upper limit (90\% CL) } \\
& & BaBar & Belle \\
\hline$\Upsilon(2 S) \rightarrow \gamma A^{0}, A^{0} \rightarrow \mu^{+} \mu^{-}$ & $0.213<m_{A^{0}}<9.37$ & - & $(0.19-8.26) \times 10^{-6}$ \\
$\Upsilon(2 S, 3 S) \rightarrow \gamma A^{0}, A^{0} \rightarrow \mu^{+} \mu^{-}$ & $0.21<m_{A^{0}}<9.3$ & $(0.3-8.3) \times 10^{-6}$ & - \\
\hline \hline
\end{tabular}

Table 4: Results of light Higgs boson searches, $\Upsilon(2 S, 3 S) \rightarrow \gamma A^{0}, A^{0} \rightarrow \mu^{+} \mu^{-}$, studied by the BaBar [10] and Belle Collaboration. The Belle result is preliminary.

identified by the photon and two charged tracks from one-prong decays of the two tau leptons. No excess is seen. The 90\% CL limits on the branching fraction are listed in Table 3 [9]. The Belle result is preliminary.

$e^{+} e^{-} \rightarrow \Upsilon(2 S, 3 S) \rightarrow \gamma A^{0}, A^{0} \rightarrow \mu^{+} \mu^{-}$

In this analysis, the signal events are reconstructed by combining a photon with a pair of oppositely-charged tracks. The charged tracks must be identified as muons by particle identification algorithms. A series of unbinned likelihood fits to the dimuon mass distribution is performed to extract the signal. No excess is seen. The $90 \%$ CL limits on the branching fraction are listed in Table 4 [10]. The Belle result is preliminary.

$e^{+} e^{-} \rightarrow \Upsilon(2 S, 3 S) \rightarrow \Upsilon(1 S) \pi^{+} \pi^{-}, \Upsilon(1 S) \rightarrow \gamma A^{0}, A^{0} \rightarrow \mu^{+} \mu^{-}$

In this analysis, the $\Upsilon(1 S)$ resonance is produced from the $\Upsilon(2 S, 3 S)$ resonance with the emission of two charged pions. The $\Upsilon(1 S)$ is identified by the dipion transition. The signal events are identified by the photon and two charged tracks. The charged tracks must be identified as muons by particle identification algorithms. A series of unbinned likelihood fits to the dimuon mass dis- 


\begin{tabular}{|c|c|cc|}
\hline \hline Mode & Mass range (GeV) & \multicolumn{2}{|c|}{ BF upper limit (90\% CL) } \\
& & BaBar & Belle \\
\hline$\Upsilon(1 S) \rightarrow \gamma A^{0}, A^{0} \rightarrow \mu^{+} \mu^{-}$ & $0.212<m_{A^{0}}<9.20$ & $(0.28-9.7) \times 10^{-6}$ & - \\
$\Upsilon(1 S) \rightarrow \gamma A^{0}, A^{0} \rightarrow \mu^{+} \mu^{-}$ & $0.212<m_{A^{0}}<9.27$ & - & $(0.01-11.86) \times 10^{-6}$ \\
\hline
\end{tabular}

Table 5: Results of light Higgs boson searches, $e^{+} e^{-} \rightarrow \Upsilon(2 S) \rightarrow \Upsilon(1 S) \pi^{+} \pi^{-}, \Upsilon(1 S) \rightarrow \gamma A^{0}, A^{0} \rightarrow \mu^{+} \mu^{-}$, studied by the BaBar [11] and Belle Collaboration. The BaBar result is formed by combining the $\Upsilon(2 S, 3 S)$ dataset. The Belle result is preliminary.

tribution is performed to extract the signal. No excess is seen. The 90\% CL limits on the branching fraction are listed in Table 5 [11]. The Belle result is preliminary.

\section{Summary}

The selected searches of $A^{0}$ decays into $\mu^{+} \mu^{-}$and $\tau^{+} \tau^{-}$final states have been studied by BaBar and Belle Collaboration. All studies show null results.

\section{Search for low-mass dark-sector Higgs bosons}

Recent results from terrestrial and satellite experiments have motivated the proposal of a new, hidden gauge sector under which WIMP-like dark matter particles are charged [12].

An Abelian gauge field, the dark photon A, couples this dark sector to Standard Model (SM) particles through its kinetic mixing with the SM hypercharge fields [13]. In this framework dark matter particles can annihilate into pairs of dark photons, which subsequently decay to SM particles. The dark photon mass is constrained to be at most a few $\mathrm{GeV}$ to be compatible with astrophysical constraints [14]. In a minimal model [15], the dark photon mass is generated via the Higgs mechanism, adding a dark Higgs boson $\mathrm{h}^{\prime}$ to the theory. The mass hierarchy between these two particles is not constrained, and the dark Higgs boson could be light as well.

The Higgsstrahlung process, $e^{+} e^{-} \rightarrow \mathrm{Ah}^{\prime}, \mathrm{h}^{\prime} \rightarrow \mathrm{AA}$, offers a gateway to the hidden gauge sector. This process is one of the few suppressed by only a single power of the mixing strength, and the background is expected to be almost negligible.

The BaBar [16] measurement is performed in the range $0.8<m_{h^{\prime}}<10.0 \mathrm{GeV}$ and $0.25<$ $m_{A}<3.0 \mathrm{GeV}$, with the constraint $m_{h^{\prime}}>2 m_{A}$. The signal is either fully reconstructed into $3\left(\ell^{+} \ell^{-}\right)$, $2\left(\ell^{+} \ell^{-}\right)\left(\pi^{+} \pi^{-}\right), \ell^{+} \ell^{-} 2\left(\pi^{+} \pi^{-}\right)$final states $(\ell=e, \mu)$ or partially reconstructed in the $2\left(\mu^{+} \mu^{-}+X\right)$ and $\mu^{+} \mu^{-} e^{+} e^{-}+X$ channels, where $\mathrm{X}$ denotes any final state other than a pair of pions or leptons.

The Belle measurement is performed in the range $0.5<m_{h^{\prime}}<10.5 \mathrm{GeV}$ and $0.25<m_{A}<3.5$ $\mathrm{GeV}$, with the constraint $m_{h^{\prime}}>2 m_{A}$. The signal is fully reconstructed into $3\left(\ell^{+} \ell^{-}\right),\left(e^{+} e^{-}\right) 2\left(\pi^{+} \pi^{-}\right)$, $2\left(\mu^{+} \mu^{-}\right) e^{+} e^{-}$final states $(\ell=e, \mu)$.

In both $\mathrm{BaBar}$ and Belle studies, no significant signal is observed. Upper limits on the $e^{+} e^{-}$ $\rightarrow \mathrm{Ah}^{\prime}, \mathrm{h}^{\prime} \rightarrow \mathrm{AA}$ cross section are set as a function of the hidden Higgs and hidden photon masses. The limits on each channels are combined to extract 90\% CL upper limits, which is at the level of 10 - $100 \mathrm{ab}$. Upper limits on the product of the mixing angle and the dark coupling constant in the case of a hidden sector with an Abelian Higgs boson have been set at the level of $10^{-10}-10^{-8}$ [17]. The Belle results are preliminary. 


\section{References}

[1] B. Aubert et al.(BABAR Collaboration), Nucl. Instrum. Meth. A 479, 1 (2002).

[2] S. Kurokawa and E. Kikutani, Nucl. Instrum. Meth. A 499, 1 (2003), and other papers included in this Volume; T.Abe et al., Prog. Theor. Exp. Phys. 2013, 03A001 (2013) and following articles up to 03A011.

[3] A. Abashian et al. (Belle Collaboration), Nucl. Instrum. Meth. A 479, 117 (2002); also see detector section in J.Brodzicka et al., Prog. Theor. Exp. Phys. 2012, 04D001 (2012).

[4] P.W. Higgs, Phys. Rev. Lett. 13, 508 (1964).

[5] G. Aad et al.(ATLAS Collaboration), Phys. Lett. B 716, 1(2012); S. Chatrchyan et al. (CMS Collaboration), Phys. Lett. B 716, 30 (2012).

[6] R. Dermisek and J. F. Gunion, Phys. Rev. Lett. 95, 041801 (2005); R. Dermisek, J. F. Gunion, and B. McElrath, Phys. Rev. D 76, 051105 (2007).

[7] F. Wilczek, Phys. Rev. Lett. 39, 1304 (1977).

[8] B. Aubert et al.(BABAR Collaboration), Phys. Rev. Lett. 103, 181801 (2009).

[9] J. P. Lees et al. (BABAR Collaboration), Phys. Rev. D 88, 071102 (2013).

[10] B. Aubert et al.(BABAR Collaboration), Phys. Rev. Lett. 103, 081803 (2009).

[11] J. P. Lees et al. (BABAR Collaboration), Phys. Rev. D 87, 031102 (2013).

[12] R. Bernabei et al., Eur. Phys. J. C 56: 333-355 (2008). M. Aguilar et al. (AMS Collaboration), Phys. Rev. Lett. 110, 141102 (2013).

[13] B. Holdom, Phys. Lett. B 166, 196 (1986).

[14] O. Adriani et al. (PAMELA Collab.), Nature 458, 607 (2009). M. Ackermann et al. (Fermi LAT Collab.), Phys. Rev. D 82, 092004 (2010).

[15] B. Batell, M. Pospelov, and A. Ritz, Phys. Rev. D 79, 115008 (2009).

[16] J. P. Lees et al. (BABAR Collaboration), Phys. Rev. Lett. 108, 211801 (2012).

[17] J. D. Bjorken, R. Essig, P. Schuster, and N. Toro, Phys. Rev. D 80, 075018 (2009). 